\title{
Note on the Transliteration System
}

I have deliberately minimized the usual transliteration acrobatics. Preference is given to Persian pronunciation of words and names Ketabkhane instead of Kitabkhana, or Daulat instead of Dawlat for which the Cambridge History of Iran has been adopted, with some modifications. Anglicized names and terms remain unchanged except when the Arabicized version has predominated and where the referent also has a proper Persian home; so ayvan instead of iwan, or Nauruz instead of Nawruz. Such terms as have entered English-language dictionaries as sultan and harem are utilized when speaking generally; but not when it is part of a proper name, such as Shah Soltan Hossayn. I have not used diacritical marks unless the proper pronunciation of the term bears significantly on the discussion. City names in common usage, such as Isfahan, remain unchanged unless in the Persian title of a book, such as Esfahan. As much as I have tried, I am certain there are inconsistencies, maybe even egregious ones, for which I beg the reader's indulgence. 